\title{
NID Copper Sample Analysis
}

Richard T Kouzes

Zihua Zhu

\section{February 2011}

\section{September 2011 Revision 1}
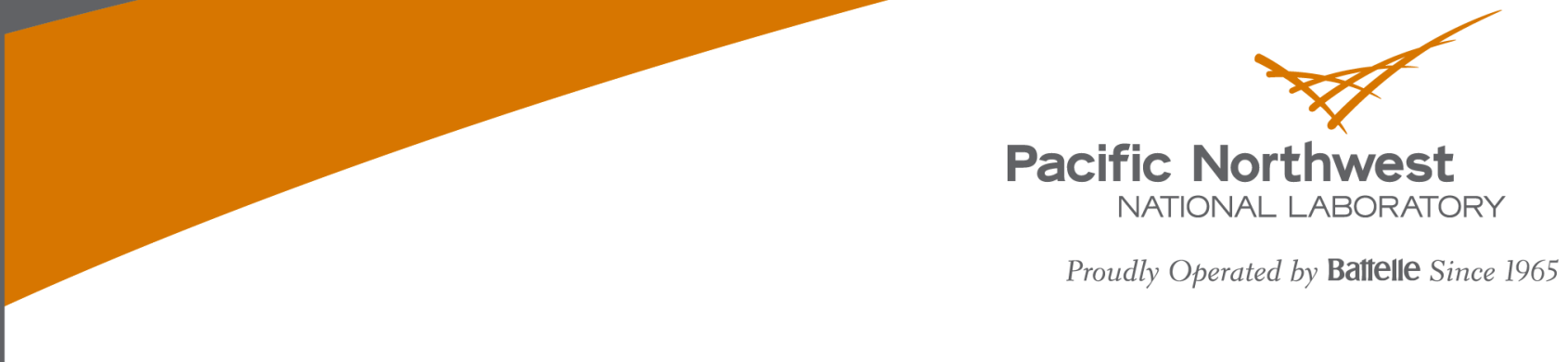


\title{
DISCLAIMER
}

This report was prepared as an account of work sponsored by an agency of the United States Government. Neither the United States Government nor any agency thereof, nor Battelle Memorial Institute, nor any of their employees, makes any warranty, express or implied, or assumes any legal liability or responsibility for the accuracy, completeness, or usefulness of any information, apparatus, product, or process disclosed, or represents that its use would not infringe privately owned rights. Reference herein to any specific commercial product, process, or service by trade name, trademark, manufacturer, or otherwise does not necessarily constitute or imply its endorsement, recommendation, or favoring by the United States Government or any agency thereof, or Battelle Memorial Institute. The views and opinions of authors expressed herein do not necessarily state or reflect those of the United States Government or any agency thereof.

\author{
PACIFIC NORTHWEST NATIONAL LABORATORY \\ operated by \\ BATTELLE \\ for the \\ UNITED STATES DEPARTMENT OF ENERGY \\ under Contract DE-AC05-76RL01830
}

Printed in the United States of America
Available to DOE and DOE contractors from the Office of Scientific and Technical Information,
P.O. Box 62, Oak Ridge, TN 37831-0062;
ph: (865) 576-8401
fax: $(865)$ 576-5728
email: reports@adonis.osti.gov

\begin{abstract}
Available to the public from the National Technical Information Service, U.S. Department of Commerce, 5285 Port Royal Rd., Springfield, VA 22161 ph: (800) 553-6847 fax: $(703) 605-6900$ email: orders@ntis.fedworld.gov online ordering: http://www.ntis.gov/ordering.htm
\end{abstract}

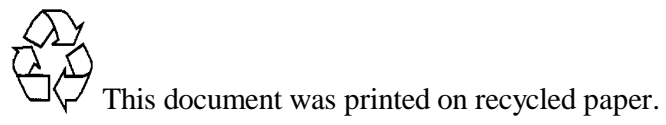


PNNL-20373

Revision 1

NID Copper Sample Analysis

Richard T Kouzes

Zihua Zhu

February 2011

September 2011 Revision 1

Pacific Northwest National Laboratory

Richland, Washington 99352 


\section{Executive Summary}

The current focal point of the nuclear physics program at PNNL is the MAJORANA DEMONSTRATOR, and the follow-on Tonne-Scale experiment, a large array of ultra-low background high-purity germanium detectors, enriched in ${ }^{76} \mathrm{Ge}$, designed to search for zeroneutrino double-beta decay $(0 v \beta \beta)$. This experiment requires the use of germanium isotopically enriched in ${ }^{76} \mathrm{Ge}$. The MAJORANA DEMONSTRATOR is a DOE and NSF funded project with a major science impact.

The Demonstrator will utilize ${ }^{76}$ Ge from Russia, but for the Tonne-Scale experiment it is hoped that an alternate technology, possibly one under development at Nonlinear Ion Dynamics (NID), will be a viable, US-based, lower-cost source of separated material. Samples of separated material from NID require analysis to determine the isotopic distribution and impurities. DOE is funding NID through an SBIR grant for development of their separation technology for application to the Tonne-Scale experiment.

The Environmental Molecular Sciences facility (EMSL), a DOE user facility at PNNL, has the required mass spectroscopy instruments for making isotopic measurements that are essential to the quality assurance for the MAJORANA DEMONSTRATOR and for the development of the future separation technology required for the Tonne-Scale experiment.

A sample of isotopically separated copper was provided by NID to PNNL in January 2011 for isotopic analysis as a test of the NID technology. The results of that analysis are reported here.

A second sample of isotopically separated copper was provided by NID to PNNL in August 2011 for isotopic analysis as a test of the NID technology. The results of that analysis are also reported here. 


\section{Acronyms and Abbreviations}

NID

PNNL
Nonlinear Ion Dynamics

Pacific Northwest National Laboratory 


\section{Contents}

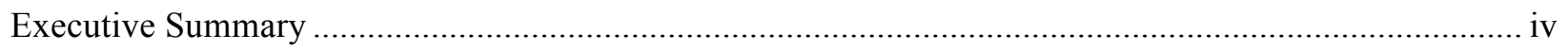

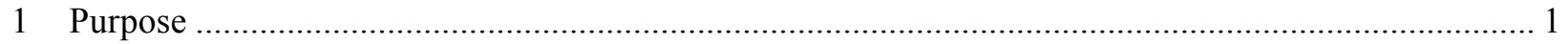

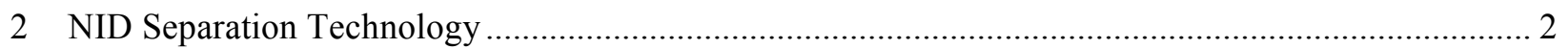

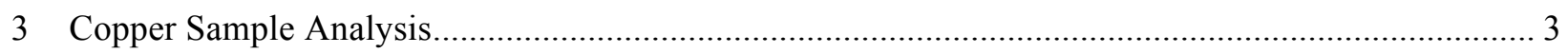

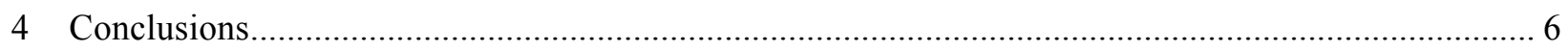

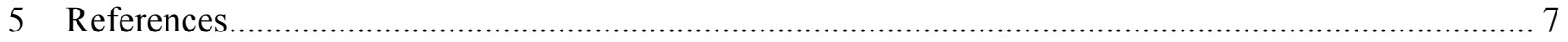




\section{Figures and Tables}

\section{Figures}

Figure 3.1. Copper sample on glass from NID. The copper coating is divided in three parts: \#1 from $\mathrm{R}=2.5 \mathrm{~cm}$ to $3.2 \mathrm{~cm}$; $\# 2$ from $\mathrm{R}=2.0 \mathrm{~cm}$ to $2.5 \mathrm{~cm}$; \#3 from $\mathrm{R}=1.7$ to $2.0 \mathrm{~cm}$.................................... 3

\section{Tables}

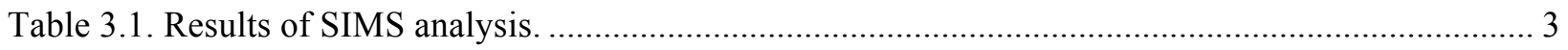




\section{Purpose}

Neutrinoless double-beta decay provides the physics community with the opportunity to build on our successes in understanding the neutrino and crafting a new standard model. With the results from Super-Kamiokande, SNO, KamLAND, and other neutrino experiments, we have demonstrated that neutrinos are massive, change flavor, and play an important role in the universe. Measuring the absolute mass of neutrinos and determining their Majorana nature are two of the most important goals of the physics community today. The standard double-beta decay process results in emission of two beta particles and two neutrinos, a process that was first reliably observed in ${ }^{76} \mathrm{Ge}$ by the PNNL-University of South Carolina collaboration in 1990. The much rarer, and yet unconfirmed, $0 v \beta \beta$ process results in emission of only the two beta particles. Observation of this process would provide direct evidence that neutrinos are Majorana particles and that lepton number is not conserved.

The MAJORANA Collaboration was initiated in 1999 in order to carry out a $0 v \beta \beta$ experiment in ${ }^{76} \mathrm{Ge}$. MAJORANA is a collaboration of about 100 scientists at 20 institutions worldwide supported by DOE Office of Science Office of Nuclear Physics, the National Science Foundation, and other international funding agencies. The current plan calls for the MAJORANA DEMONSTRATOR to be constructed and operated over the next several years in parallel with the European ${ }^{76} \mathrm{Ge}$ experiment (GERDA). This project is proposed to be followed by a single, merged, Tonne-Scale, international experiment for $0 v \beta \beta$ in ${ }^{76} \mathrm{Ge}$.

The Majorana Demonstrator experiment, the predecessor to a Tonne-Scale experiment, is now constructing an essentially background-free measurement of $0 v \beta \beta$ in $20 \mathrm{~kg}$ of natural Ge plus $20 \mathrm{~kg}$ of ${ }^{76} \mathrm{Ge}$ with the goal of determining lepton number conservation and the viability of a Tonne-Scale experiment. The MAJORANA DEMONSTRATOR requires $\sim 30 \mathrm{~kg}$ of isotopically enriched ${ }^{76} \mathrm{Ge}$. Currently, the only source of enriched ${ }^{76} \mathrm{Ge}$ is from Russia, at a cost of $\sim \$ 85 / \mathrm{g}$. This material will be delivered to the MAJORANA DEMONSTRATOR experiment in FY11-FY12, with quality assurance (QA) samples arriving on a periodic basis during this time. These QA samples will require precision isotopic evaluation. One such measurement was performed in 2009 under an EMSL rapid proposal on a single sample using SIMS instruments [Elliott 2009]. Further measurements will take place during FY11-FY12 at EMSL. 


\section{NID Separation Technology}

Stable isotopes are widely used in scientific research, medical diagnostics \& treatment, as well as nuclear, semiconductor and other industries. Large quantities of separated isotopes are needed for rare decay detection in nuclear physics research such as the MAJORANA DEMONSTRATOR and the proposed Tonne-Scale experiment. Nonlinear Ion Dynamics (NID) was awarded a SBIR Phase II grant by DOE to develop an Integrated Spin System (ISS) for production of large quantities of stable isotopes in support of MAJORANA. PNNL has been working with NID on the development of their ISS capability, representing the MAJORANA Collaboration.

The ISS is an outgrowth of NID experience with Ion Cyclotron Resonance Heating (ICRH) technique for isotope separation. The ISS approach uses a rapidly rotating plasma in a strong magnetic field to drive high density $\left(10^{23} / \mathrm{m}^{3}\right)$ neutral particles to rotate at high speed (up to 100,000 revolutions per second). Isotopes with different masses are separated by the plasma's "centrifugal" force. The ISS approach will use raw germanium as the feedstock and the separated ${ }^{76} \mathrm{Ge}$ product is deposited in solid form. In comparison, traditional gaseous centrifuge requires a gaseous Ge compound as the working medium, and several chemical processes are involved that can introduce impurities into the final product. Moreover, since the raw Ge material does not need to be ionized in the ISS, this approach can potentially separate the large quantities of ${ }^{76} \mathrm{Ge}$ required for the Tonne-Scale Ge experiment with much less power usage than conventional approaches, including gaseous centrifuges in Russia, and the ICRH method. This method has been demonstrated for noble gases by NID. Work has begun under the DOE grant on Ge separation, and analysis of the isotopic enrichment of this method is required. The hope for developing this US-based lower-cost alternative to the Russian centrifuge approach is the basis for the DOE support of this work at NID.

Secondary ion mass spectrometry (SIMS) systems at EMSL are used for the benchmark isotopic analysis of samples produced by the ISS system at NID, as well as the samples from Russia. The sample analysis results from the SIMS at EMSL will feedback to the development of the ISS system. Enriched Ge and other samples in the form of thin films (a few microns in thickness) deposited on silicon or glass substrates are sent to the PNNL SIMS team by NID for analysis. 


\section{First Copper Sample Analysis}

A sample of isotopically separated copper was provided by NID for analysis in late January 2011. This sample, collected in $15 \mathrm{~s}$, consisted of three stripes on a glass plate, as seen in Figure 3.1. Natural copper consists of two isotopes, ${ }^{63} \mathrm{Cu}(69.17 \%)$ and ${ }^{65} \mathrm{Cu}(30.83 \%)$. The sample was analyzed on an EMSL SIMS instrument.

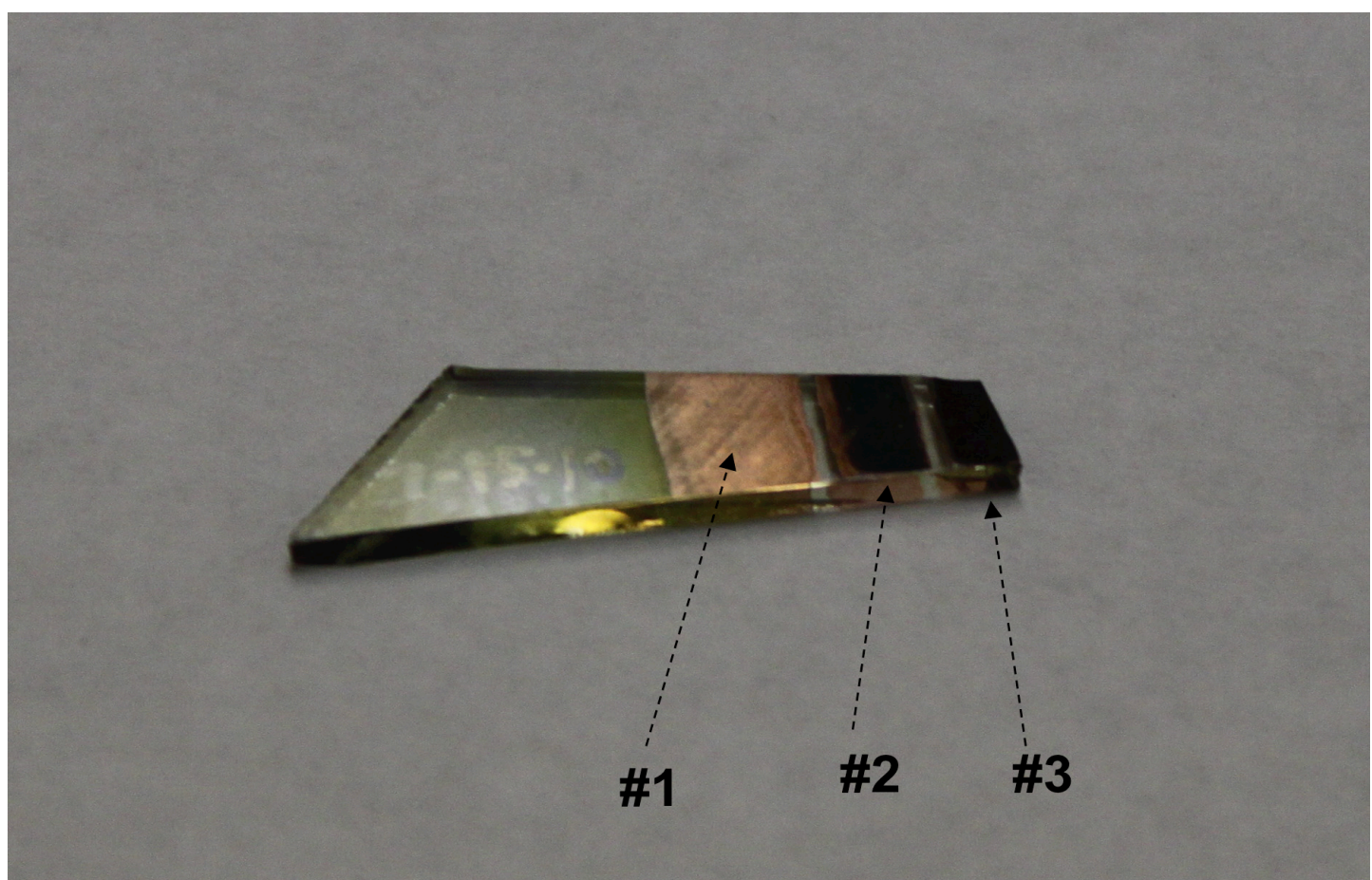

Figure 3.1. Copper sample on glass from NID. The copper coating is divided in three parts: \#1 from $\mathrm{R}=2.5 \mathrm{~cm}$ to $3.2 \mathrm{~cm}$; $\# 2$ from $\mathrm{R}=2.0 \mathrm{~cm}$ to $2.5 \mathrm{~cm}$; $\# 3$ from $\mathrm{R}=1.7$ to $2.0 \mathrm{~cm}$

The result of the SIMS measurements are shown in Table 3.1. It is seen that the reference material agrees with the documented value for copper isotopics. The isotopic values vary for the three sample locations. The largest enhancement in isotopic ratio is seen at Location \#1, where the ${ }^{65} \mathrm{Cu}$ was enhanced from 0.31 in natural copper to 0.39 in the enriched sample, and the ${ }^{63} \mathrm{Cu}$ was depleted in proportion. Location \#2 showed little enrichment, while location \#3 showed some depletion in ${ }^{65} \mathrm{Cu}$.

Table 3.1. Results of SIMS analysis.

\begin{tabular}{|l|l|l|}
\hline & ${ }^{63} \mathrm{Cu}$ Ratio & ${ }^{65} \mathrm{Cu}$ Ratio \\
\hline Document value & 0.6917 & 0.3083 \\
\hline My reference (a Cu grid) & $0.693+/-0.008$ & $0.307+/-0.008$ \\
\hline Location 1 & 0.615 & 0.385 \\
\hline Location 2 & 0.677 & 0.323 \\
\hline Location 3 & 0.729 & 0.271 \\
\hline
\end{tabular}


NID plans to collect using a cooled metallic surface in their next runs to ensure more samples are collected for a longer time and that the "layering" effect would not be strong. With the glass collectors they found that the upper layer had a much better isotopic concentration, greater than $70 \%$. However the layers below have smaller concentrations. The layer dependence is due to the temporal operation. The later time tends to give a better separation. 


\section{Second Copper Sample Analysis}

A second set of samples of isotopically separated copper was provided by NID for analysis in late August 2011. These samples consisted of small flakes, as seen in Figure 4.1. Four samples were provided, but only three of them were usable for analysis. Natural copper consists of two isotopes, ${ }^{63} \mathrm{Cu}(69.17 \%)$ and ${ }^{65} \mathrm{Cu}(30.83 \%)$. The sample was analyzed on an EMSL SIMS instrument.

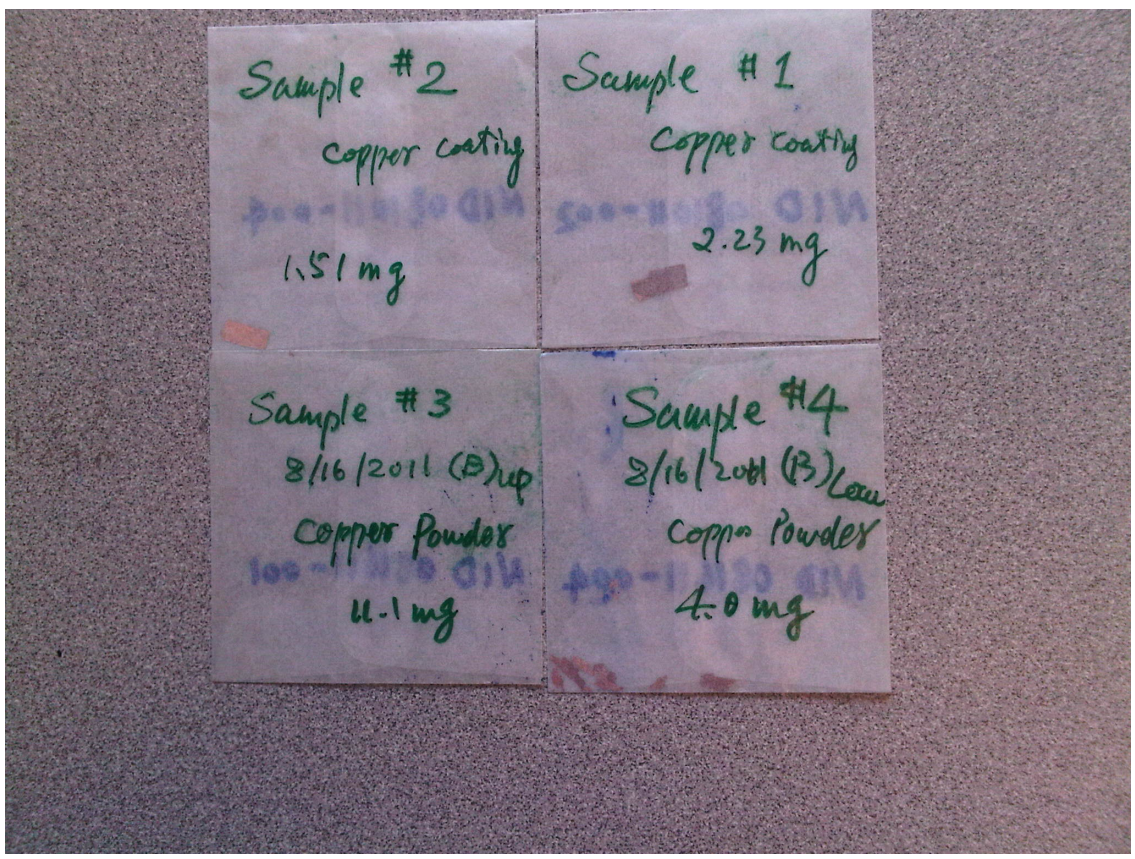

Figure 4.1. Four copper samples in bags from NID.

The results of the SIMS measurements are shown in Table 4.1. The isotopic values were measured on both sides of the samples. The samples showed only a slight increase in ${ }^{63} \mathrm{Cu}$ over what is expected from a natural sample.

Table 4.1. Results of SIMS analysis.

\begin{tabular}{|l|l|l|}
\hline & \multicolumn{1}{|c|}{${ }^{\mathbf{6 3}} \mathbf{C u ~ \%}$} & \multicolumn{1}{c|}{${ }^{\mathbf{6 5}} \mathbf{C u ~ \%}$} \\
\hline Natural & 0.6917 & 0.3083 \\
\hline 1\# dark side & $0.710 \pm 0.007$ & $0.290 \pm 0.007$ \\
\hline 1\# shining side & $0.712 \pm 0.006$ & $0.288 \pm 0.006$ \\
\hline 2\# dark side & $0.706 \pm 0.007$ & $0.294 \pm 0.007$ \\
\hline 2\# shining side & $0.707 \pm 0.005$ & $0.293 \pm 0.005$ \\
\hline 4\# dark side & $0.706 \pm 0.005$ & $0.294 \pm 0.005$ \\
\hline 4\# shining side & $0.702 \pm 0.005$ & $0.298 \pm 0.005$ \\
\hline
\end{tabular}

NID plans to continue development of their technique. 


\section{Conclusions}

The results of the isotopic enrichment of copper by NID using their plasma centrifuge shows an enrichment of about $25 \%$ of the lower abundance copper isotope, ${ }^{65} \mathrm{Cu}$. For application to enrichment of large amounts of material, higher enrichments are desired. It is anticipated that NID will provide further samples for isotopic measurement. 


\section{References}

S.R. Elliott, V.E. Guiseppe, B.H. LaRoque, R.A. Johnson, S.G. Mashnik, Fast-Neutron Activation of Long-Lived Isotopes in Enriched Ge, submitted for publication December 2009. 


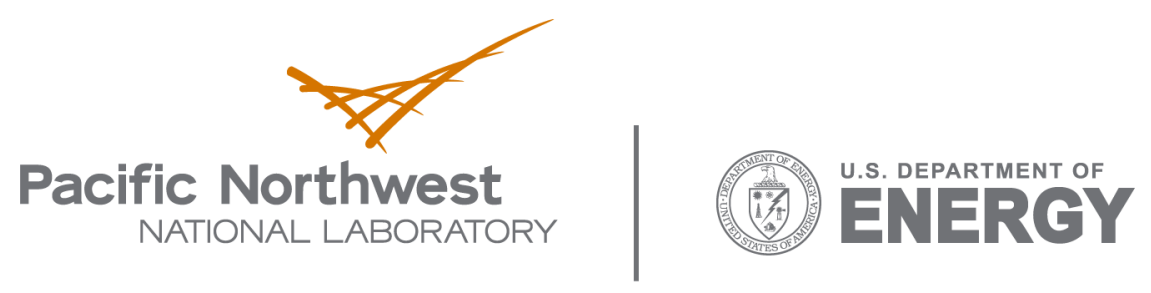

902 Battelle Boulevard

P.O. Box 999

Richland, WA 99352

1-888-375-PNNL (7665)

www.pnl.gov 